\title{
Modulation of Interparticle Gap for Enhanced SERS Sensitivity in Chemically Stable Ag@Au Hetero-Architectures
}

\author{
Chunfang $\mathrm{Wu}^{\mathrm{a}, \mathrm{b}, *}$, Qing $\mathrm{Hu}^{\mathrm{a}}$, Melissa Benison ${ }^{\mathrm{b}}$, Karen Faulds $^{\mathrm{b}}$, Duncan Graham ${ }^{\mathrm{b}}$ \\ a School of Optoelectronic Engineering, Xi'an Technological University, Xi'an, 710021, P.R.China \\ ${ }^{b}$ Department of Pure and Applied Chemistry, University of Strathclyde, Glasgow, G1 1RD, UK
}

\section{Abstract:}

Despite excellent surface-enhanced Raman scattering (SERS) activity, the poor chemical stability of Ag nanoparticles severely hinders their application as SERS substrates. In this paper, a two-step process was employed to prepare highly sensitive and chemically stable Ag@Au hetero-architectures, in situ growth of $\mathrm{Ag}$ nanoparticles on a $\mathrm{Si}$ wafer followed by Au coating through ion sputtering. Owing to the chemical inertness of $\mathrm{Au}$, the $\mathrm{Ag} @ \mathrm{Au}$ hetero-architecture substrates exhibited enhanced chemical stability compared to pure Ag nanoparticle substrates. In particular, the deposition of $\mathrm{Au}$ coatings allows efficient modulation of the gap between neighboring $\mathrm{Ag}$ nanoparticles and strengthens the surface plasmon coupling effect. As a consequence, the Ag@Au hetero-architecture substrates exhibited higher SERS sensitivity than pure Ag nanoparticle substrates. Furthermore, a possible mechanism for the enhanced SERS sensitivity of the Ag@Au hetero-architecture substrates was proposed and discussed. The present work proposes an effective and facile way to tune the SERS sensitivity and chemical stability of Ag nanoparticle substrates, and furthermore provides a promising application of SERS in oxidative environments or biological systems .

\section{Keywords:}

SERS; Ag@Au; Hetero-architecture; Chemical stability; Highly sensitive;

* Corresponding author.

E-mail address: $\quad \underline{\text { wuchf@xatu.edu.cn }}$ 


\section{Introduction}

Surface-enhanced Raman scattering (SERS) is a highly sensitive analytical tool for the detection of trace amounts of analytes. The use of noble metal nanoparticles yields significant enhancement in Raman scattering intensity as a result of both chemical and electromagnetic enhancement mechanisms. ${ }^{1,2}$ The heightened electric field experienced by molecules adsorbed, or in close proximity to the surface of metal nanoparticles results from excitation of the nanoparticle's localized surface plasmon resonance (LSPR). Interaction between the adsorbed species and the nanoparticle's polarized electron cloud provides the dominant contribution to the enhancement of the Raman signal intensity. Ag nanoparticles are widely used because of their excellent SERS activity. However, their practical application in oxidizing environments is hindered by their poor chemical stability. In contrast, Au nanoparticles have better chemical stability, but lower SERS enhancement capabilities than Ag nanoparticles of a similar size. ${ }^{3}$ As a result, many studies have tried to improve the stability of $\mathrm{Ag}$ nanoparticles by forming Ag-Au alloys ${ }^{4-6}$ or Ag@ Au core-shell structures. ${ }^{7-11}$ The surface plasmon resonance (SPR) of $\mathrm{Ag}-\mathrm{Au}$ alloy nanoparticles can be tuned to fall between that of $\mathrm{Ag}$ nanoparticles and that of Au nanoparticles based on the Ag to Au atomic ratio. In contrast, the LSPR of Ag@Au core-shell nanoparticles can be shifted to longer wavelengths, such as in the near-infrared region. Furthermore, the presence of $\mathrm{Au}$ in the $\mathrm{Ag} @ \mathrm{Au}$ nanoparticles, whether as an alloy or as a shell, yields improved chemical stability compared to Ag nanoparticles alone.

The galvanic replacement reaction (GRR) between $\mathrm{Ag}$ and $\mathrm{AuCl}_{4}{ }^{-}$has been previously used to form $\mathrm{Ag}-\mathrm{Au}$ alloys and $\mathrm{Ag} @ \mathrm{Au}$ core-shell nanoparticles, with only minor differences reported between multiple studies. ${ }^{4-11}$ In some works, ${ }^{7,8} \mathrm{Ag}$ nanoparticles were coated with an Au shell by simply placing the nanoparticles in a $\mathrm{KAuCl}_{4}$ or $\mathrm{HAuCl}_{4}$ solution with heating. However, others ${ }^{4,11}$ reported that the addition of a reductant, such as $\mathrm{NaBH}_{4}$, trisodium citrate, or $\mathrm{NH}_{2} \mathrm{OH}$ and $\mathrm{HCl}$ in a $\mathrm{HAuCl}_{4}$ solution, ${ }^{10}$ was necessary to form an Au coating on Ag nanoparticles. Although thin shells of $\mathrm{Au}$ have been successfully produced on separate Ag nanoparticles in solution using the GRR, ${ }^{12,13}$ a solid substrate, such as closely packed Ag nanoparticles on Si wafer, cannot be coated with an Au film using this method. Thus, a new approach for preparing stable SERS substrates composed of closely packed Ag@Au hetero-architectures would be valuable for applications in which SERS 
measurements need to be taken in corrosive or harsh chemical environments.

The proposed new approach involved two steps, and yielded highly sensitive and chemically stable Ag@Au hetero-architectures on Si wafer. Following Ag nanoparticle growth on the Si wafer, ion sputtering was used to deposit thin films of $\mathrm{Au}$ over the localized Ag nanoparticles. By modulating the sputtering time, Au films of different thicknesses were deposited onto the immobilized Ag nanoparticles, and the relationship between sensitivity and stability of the different $\mathrm{Ag} @ \mathrm{Au}$ hetero-architecture substrates on Au film thickness was carefully evaluated. The results and analysis showed that Ag@Au hetero-architecture substrates exhibited not only excellent chemical stability, but also higher SERS sensitivity than pure Ag nanoparticle substrates. Finally, a possible mechanism for the enhanced SERS sensitivity was proposed and discussed.

\section{Experimental Section}

Si wafers with one polished side were purchased from Zhongjing Keyi, Beijing, China. Silver nitrate (>99\%), $\mathrm{NaBH}_{4}$, (3-Mercaptopropyl) trimethoxysilane (MPTMS), ascorbic acid and Rhodamine 6G (R6G) (99\%) were purchased from Sigma-Aldrich Co., Ltd. Deionized water with a resistance of $18.25 \mathrm{M} \Omega \bullet \mathrm{cm}$ was used throughout this work.

The synthetic route for the preparation of $\mathrm{Ag} @ \mathrm{Au}$ hetero-architectures is schematically illustrated in Fig.1. Briefly, the Si wafer was firstly soaked in a mixture of $\mathrm{NH}_{3} / \mathrm{H}_{2} \mathrm{O}_{2} /$ water $(1: 1: 5 v / v)$ and $\mathrm{HCl} / \mathrm{H}_{2} \mathrm{O}_{2} /$ water $(1: 1: 5 \mathrm{v} / \mathrm{v})$ for $15 \mathrm{~min}$ so as to complete hydroxylation of its surface. After being rinsed and dried, the activated wafer was immediately immersed in a $1 \% v / v$ solution of MPTMS in toluene overnight to anchor MPTMS on the Si surface (silanization process). Upon immersion of the silanized $\mathrm{Si}$ wafer in a $0.25 \mathrm{mM} \mathrm{AgNO} 3$ aqueous solution for $6 \mathrm{~h}, \mathrm{Ag}^{+}$ions were absorbed on the wafer through the MPTMS bridge ( $\mathrm{Ag}^{+}$absorption process). Subsequently a layer of $\mathrm{Ag}$ atoms was formed and anchored firmly on the $\mathrm{Si}$ wafer surface by dipping the $\mathrm{Ag}^{+} / \mathrm{Si}$ wafer into a $0.05 \mathrm{M}$ $\mathrm{NaBH}_{4}$ aqueous solution for $30 \mathrm{~min}$. The formation of the activated layer of $\mathrm{Ag}$ atoms on the $\mathrm{Si}$ wafer is crucial to enabling the nucleation and growth of $\mathrm{Ag}$ nanoparticles. The final step in the formation of $\mathrm{Ag}$ nanoparticles involved immersion of the $\mathrm{Ag} / \mathrm{Si}$ wafer in a $0.25 \mathrm{mM} \mathrm{AgNO} \mathrm{Aqueous}_{3}$ solution to which a $0.5 \mathrm{mM}$ solution of ascorbic acid wad added dropwise to promote growth, distribution and capping of the Ag nanoparticles on the wafer surface. Ag nanoparticle size and interparticle gap can be tuned by repeating the final growth step as required to obtain the desired substrate morphology. Finally, thin films of Au of $\sim 4 \mathrm{~nm}, \sim 8 \mathrm{~nm}$ and $\sim 12 \mathrm{~nm}$ were deposited over the 
anchored Ag nanoparticles by ion sputtering (MCI000 ion Sputter, Hitachi). Au film thickness was calculated based on a sputtering speed of $4 \mathrm{~nm} / \mathrm{min}$ (as recommended by the manufacturer based on the discharge current) and on the sputtering time.Ag@Au4,Ag@Au8 and Ag@Au12 are abbreviations for Ag@Au hetero-architectures with Au films of $\sim 4 \mathrm{~nm}, \sim 8 \mathrm{~nm}$ and $\sim 12 \mathrm{~nm}$, respectively. The samples were characterized by field emission scanning electron microscopy (FE-SEM, Zeiss Gemini 500) to gain insight into substrate morphology. The average nanoparticle size was statistically determined from the SEM images using "Nano Measurer 1.2" software. Briefly, the software was used to obtain the size of more than two hundred nanoparticles on the Si wafer, picked from a $1 \times 0.8 \mu \mathrm{m}$ section of the SEM images for each sample, and the average particle size was calculated for each substrate. The chemical composition of the Ag@Au hetero-architecture substrates was determined using energy dispersive X-ray spectroscopy (EDX).

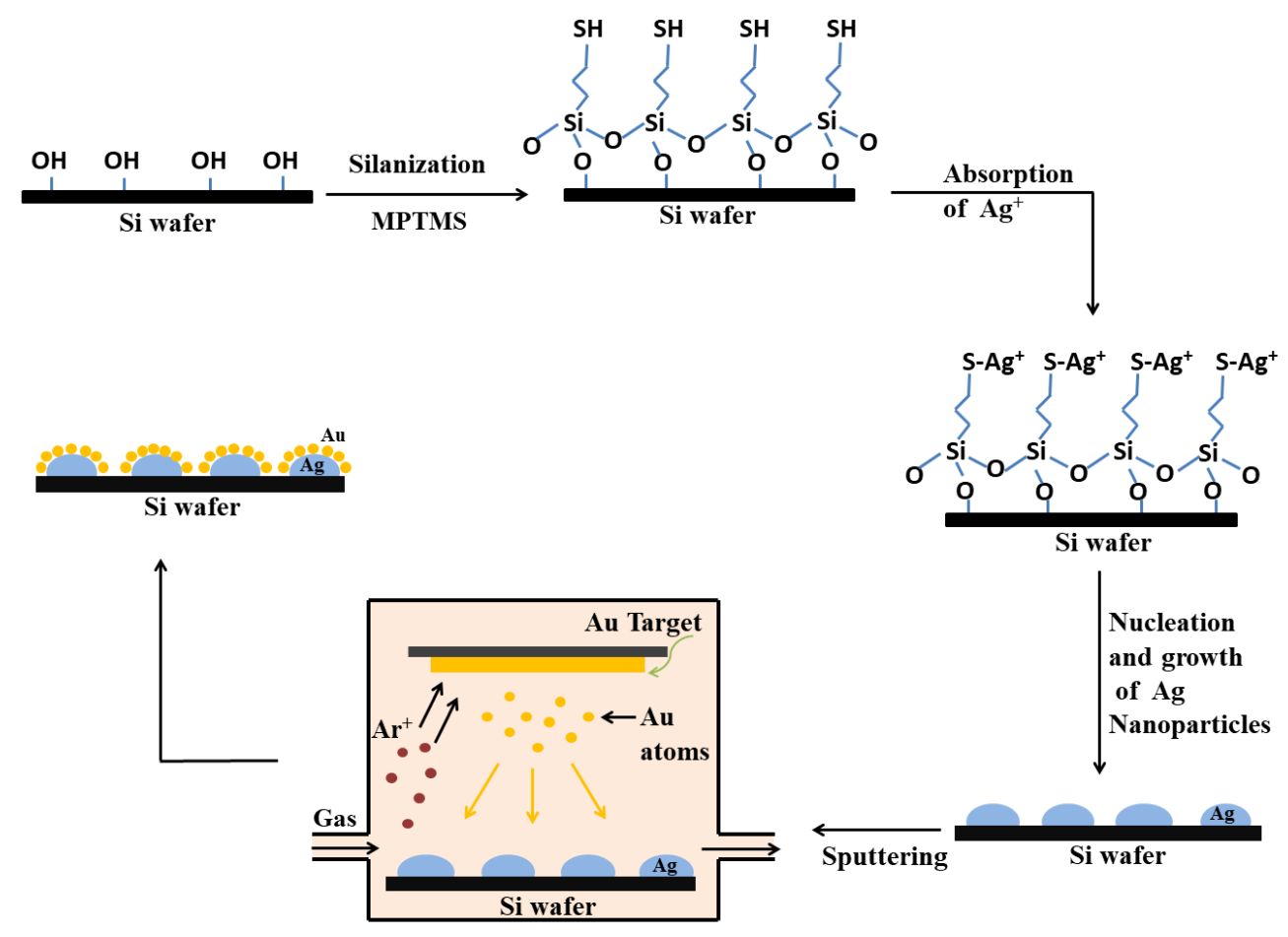

Fig.1 Schematic illustration of the synthesis of Ag@Au hetero-architectures on Si wafer.

As a bioanalytical method, SERS has been applied to areas such as chemical sensing, medical diagnostics, and the study of living cells and bacteria, as well as in immunoassays. By virtue of the chemical stability, biocompatibility and sensitivity, the as-prepared Ag@Au substrates could be used to detect a large number of substances occurring in biological systems, such as thiophenol and p-aminothiophenol, ${ }^{6}$ pyridine $^{11}$ and thiram ${ }^{4}$ or bacteria such as Escherichia Coli, Salmonella, 
Typhimurium and Bacillus Subtilis. ${ }^{14}$ In order to highlight the sensitivity and stability of the Ag@Au hetero-architecture SERS substrates, R6G was chosen as an example probe molecule and the substrates were tested using solutions of R6G to evaluate and compare their SERS properties. Aqueous solutions of R6G were prepared at concentrations of $1 \times 10^{-3}, 1 \times 10^{-5}, 1 \times 10^{-7}, 1 \times 10^{-9}$, and $1 \times 10^{-11} \mathrm{M}$. To obtain the conventional Raman spectrum of R6G, $5 \mu \mathrm{L}$ of a $1 \times 10^{-3} \mathrm{M}$ solution was drop-coated onto the surface of a pure Si wafer. For the SERS measurements, $5 \mu \mathrm{L}$ of an aqueous R6G solution of different concentrations ranging from $10^{-7}$ to $10^{-11} \mathrm{M}$ were drop-coated on the pure Ag nanoparticle substrate, and on the Ag@Au hetero-architecture substrates (Ag@Au4, Ag@Au8 and Ag@Au12). Following solvent evaporation under ambient conditions, a spot with a diameter of $\sim 5 \mathrm{~mm}$ was obtained on each substrate. A confocal Raman microscope (LabRAM HR Evolution, Horiba Jobin Yvon) was used to collect the Raman spectra. Conventional Raman and SERS spectra were obtained using an excitation wavelength of $532 \mathrm{~nm}$, an acquisition time of $1 \mathrm{~s}$, and laser power of $1 \mathrm{~mW}$. Conventional Raman and SERS spectra were collected from three randomly selected sites within the central region $\left(1 \times 1 \mathrm{~mm}^{2}\right)$ of the dried $\mathrm{R} 6 \mathrm{G}$ spot on each substrate, and the results were averaged.

\section{Results and Discussion}

\subsection{Composition and morphology}

After repeating the growth process shown in Fig. 1 four times, Ag nanoparticles with an average size of $\sim 26 \mathrm{~nm}$ were densely and homogeneously grown on the Si wafer, as shown in Fig. 2(a). The morphology of the Ag@Au hetero-architectures with Au films of 4, 8 and 12 nm is shown in Fig. 2(b), (c) and (d), respectively. Following Au film deposition, the surface of the Ag nanoparticles became roughened and the average particle diameter of the Ag@Au hetero-architectures gradually increased, from 29nm, to 31nm and 38nm for Ag@Au4, Ag@Au8 and Ag@Au12, respectively. The SEM images in Fig. 2(a-d) further indicate that the interparticle gap decreased with increasing Au film thickness. Magnified SEM images for all samples are shown in Fig. S1 (ESI). These clearly display the increase in Au film thickness accompanied by a reduction in the average interparticle gap in the Ag@Au hetero-architectures as the thickness of the Au film increased from 4 to 12 nm. 

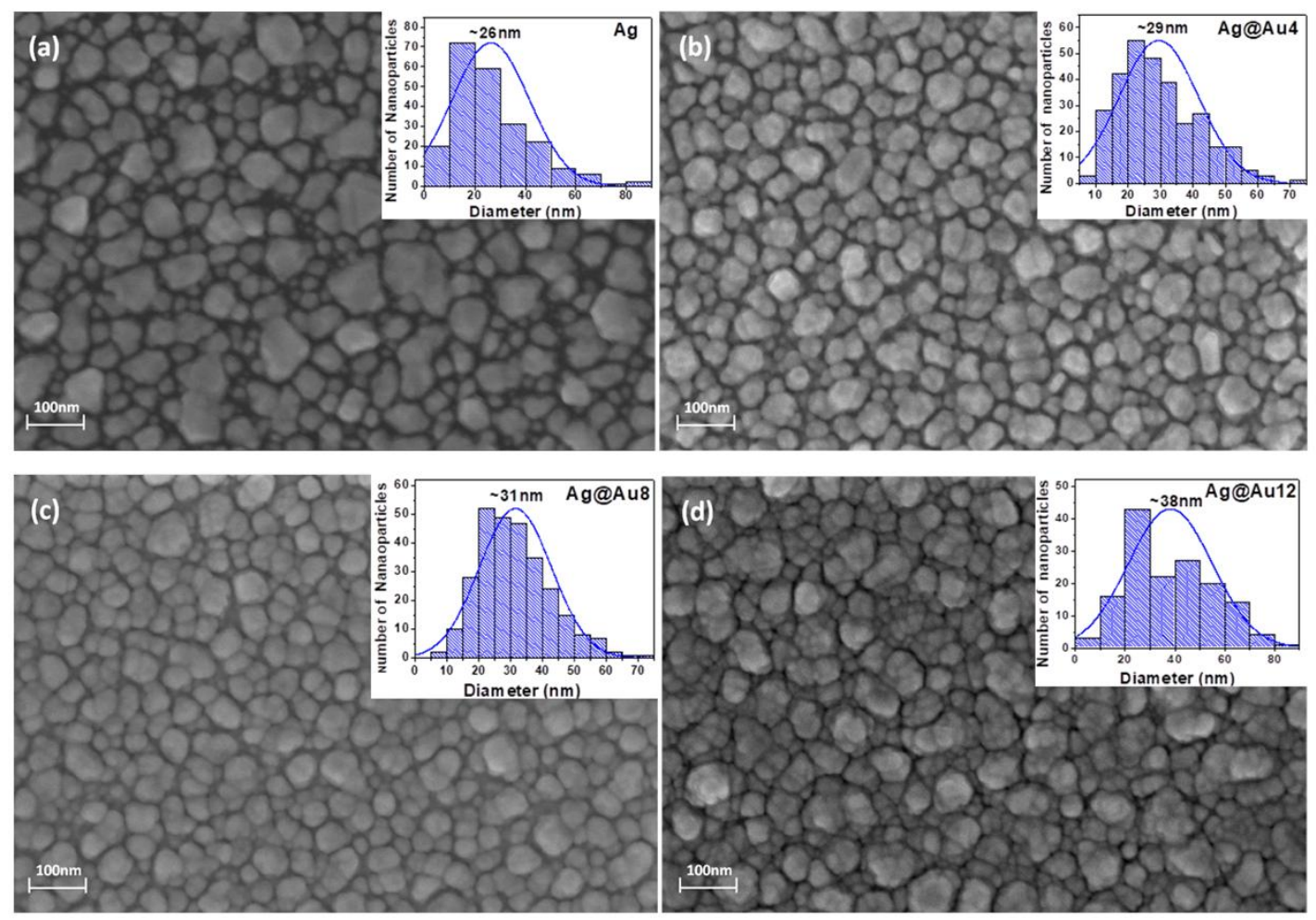

Fig.2SEM images of the substrates (a)Ag, (b)Ag@Au4, (c)Ag@Au8 and (d)Ag@Ag12. Inserts are the statistical determinations of the average nanoparticle diameters.

The average interparticle gaps between adjacent particles for different $\mathrm{Ag} @ \mathrm{Au}$ hetero-architecture samples are summarized in Table 1. It should be noted that the statistical determination of the average interparticle gap for sample Ag@Au12 was not performed due to the insufficient number of gaps resulting from nanoparticle coalescence when thicker Au films were prepared. Nonetheless, Table 1 displays a clear trend of decreasing average interparticle gap with increasing Au film thickness for Ag@Au hetero-architectures. Modulating the distance between two adjoining nanoparticles in a SERS substrate is crucial as the size of the gap strongly influences the strength of localized electromagnetic fields at the nanoparticle surface. The relationship between interparticle gap, and strength of the electromagnetic field on the SERS intensity of noble metal nanoparticle substrates will be discussed later. 
Table 1 Average interparticle gap between two adjacent particles on the different SERS substrates

\begin{tabular}{ccccc}
\hline \hline & $\mathrm{Ag}$ & $\mathrm{Ag} @ \mathrm{Au} 4$ & $\mathrm{Ag} @ \mathrm{Au} 8$ & $\mathrm{Ag} @ \mathrm{Au} 12$ \\
\hline Gap (nm) & $10.6 \pm 1.3$ & $9.8 \pm 1.1$ & $7.4 \pm 1.0$ & --- $^{\mathrm{N}}$ \\
\hline \hline
\end{tabular}

N:Unable to obtain data.

To confirm the chemical composition of these four SERS substrates, EDX spectra were collected and are shown in Fig. 3. Fig. 3(a) shows the EDX spectrum of Ag nanoparticles on a Si wafer, displaying an intense peak at $1.74 \mathrm{keV}$ for $\mathrm{Si}$, as well as typical peaks at $0.30 \mathrm{keV}$ and $3.0 \mathrm{keV}$ pertaining to Ag. The EDX spectra of the hetero-architecture substrates with Au film thicknesses of $\sim 4, \sim 8$ and $\sim 12 \mathrm{~nm}$ all contain the characteristic Au peak as shown in Fig. 3(b), (c) and (d) respectively, clearly confirming the presence of Au alongside Si and Ag. Additionally, the relative $\mathrm{Au}$ peak intensity steadily increases with respect to that of Ag for samples Ag@Au4 to Ag@Au12. This finding is consistent with the observed increase in nanoparticle size and decrease in the average interparticle gap as the thickness of the Au film is increased through successive Au sputtering cycles. The EDX spectra in Fig. 3 were also used to estimate the mass and atomic ratios of $\mathrm{Au} / \mathrm{Ag}$ in the prepared SERS substrates, as summarized in Table 2 . The data clearly shows an increase in both the mass ratio and atomic ratio of $\mathrm{Au} / \mathrm{Ag}$ in the $\mathrm{Ag} @ \mathrm{Au}$ hetero-architecture substrates as the thickness of the Au film grows from $\sim 4$ to $\sim 12 \mathrm{~nm}$. This further confirmed the presence of the Au film over the Ag nanoparticles on each sample, and that the concentration of Au in each SERS substrate had increased as a result of the extended sputtering time required to produce thicker films. 

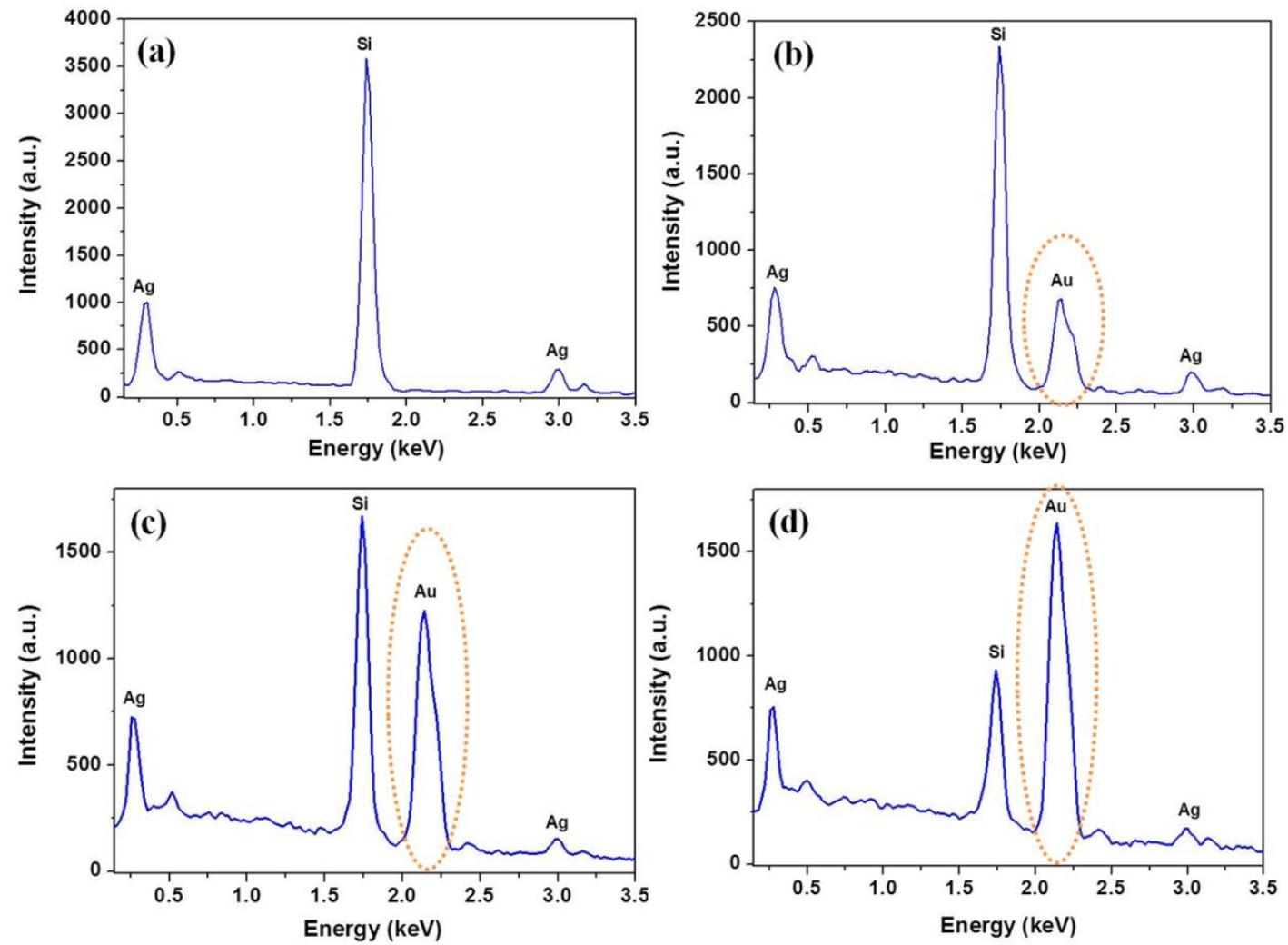

Fig.3 EDX spectra of (a)Ag, (b)Ag@Au4, (c)Ag@Au8 and (d)Ag@Au12 nanoparticles on Si wafers.

Table 2 Mass ratio and atomic ratio of Au/Ag on the different SERS substrates

\begin{tabular}{ccccc}
\hline \hline & $\mathrm{Ag}$ & $\mathrm{Ag} @ \mathrm{Au} 4$ & $\mathrm{Ag} @ \mathrm{Au} 8$ & $\mathrm{Ag} @ \mathrm{Au} 12$ \\
\hline Mass ratio $(\mathrm{Au} / \mathrm{Ag})$ & 0 & 1.45 & 4.89 & 6.96 \\
Atomic ratio $(\mathrm{Au} / \mathrm{Ag})$ & 0 & 0.79 & 2.68 & 3.81 \\
\hline \hline
\end{tabular}

\subsection{SERS spectra}

In order to quantitatively estimate the effect of the Au particle film thickness on the SERS intensity of the Ag@Au hetero-architectures, the SERS activity of pure Ag nanoparticles on Si wafer and that of the Au-coated Ag nanoparticle substrates shown in Fig.2, was compared by using $5 \mu \mathrm{L}$ of an aqueous solution of R6G at different concentrations $\left(10^{-7}, 10^{-9}\right.$, and $\left.10^{-11} \mathrm{M}\right)$ as a probe molecule. The SERS spectra, obtained by drop-coating solutions of R6G of different concentrations on the four SERS substrates, are shown in Fig. 4. The characteristic peaks of R6G at 606, 768, 1357, 1504, and $1648 \mathrm{~cm}^{-1}$, labelled as asterisks in Fig. 4, are clearly visible for all tested concentrations, on all SERS substrates. As expected, a reduction in the SERS signal intensity from all tested 
substrates was observed as the concentration of the R6G solution decreased. Nonetheless, even at the lowest tested concentration of $10^{-11} \mathrm{M}$, the typical spectrum of R6G was obtained from all SERS substrates, with minimal peak shifting. Furthermore, the position of the R6G peaks originating from specific modes of the molecule obtained from pure $\mathrm{Ag}$ nanoparticles on $\mathrm{Si}$ wafer and from the $\mathrm{Ag} @ \mathrm{Au}$ hetero-architecture substrates are in good agreement with previous reports by other groups. ${ }^{15,16}$ The excellent sensitivity displayed by both the Ag nanoparticles on Si wafer and by the $\mathrm{Ag} @ \mathrm{Au}$ hetero-architecture substrates leads us to believe they will be ideal candidates for trace analyte detection from a variety of samples. Closer analysis of the SERS spectra indicated that for pure $\mathrm{Ag}$ nanoparticles on $\mathrm{Si}$ wafer, the intensity of the peak at $606 \mathrm{~cm}^{-1}$ caused by the $\mathrm{C}-\mathrm{C}-\mathrm{C}$ ring in-plane vibration of $\mathrm{R} \mathrm{G}^{15,16}$ was $~ 4600$ counts after background subtraction when a $10^{-7} \mathrm{M}$ solution of R6G was spotted. At the same R6G concentration, the intensity of the $606 \mathrm{~cm}^{-1}$ peak was enhanced to 4900 and 5600 counts for hetero-architecture samples Ag@Au4 and Ag@Au8, respectively. Conversely, for substrate- Ag@Au12 the peak intensity reduced to 1400 counts.
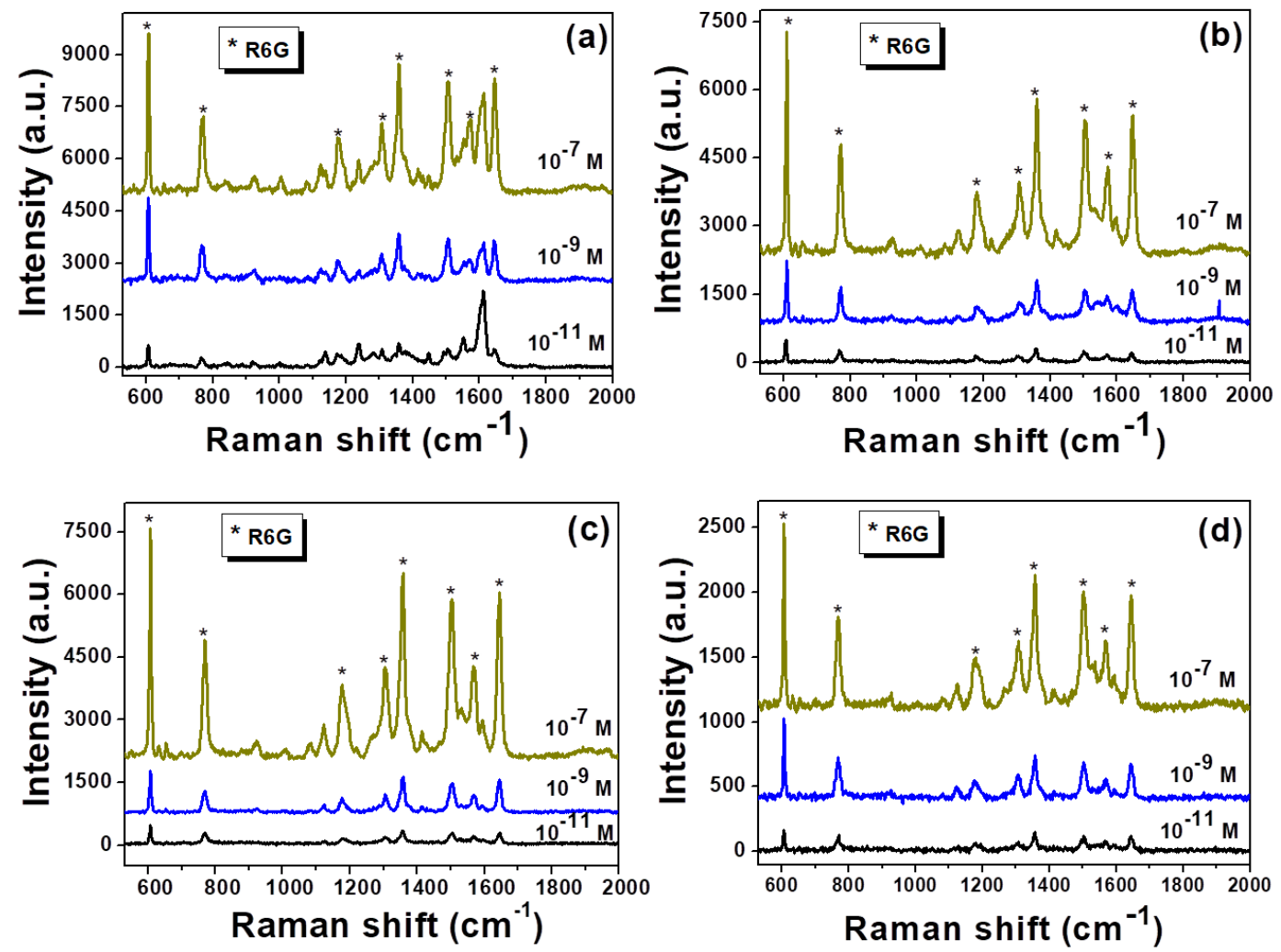

Fig.4SERS spectra of R6G drop-coated on (a)Ag, (b)Ag@Au4, (c)Ag@Au8 and (d)Ag@Au12 at three different concentrations: $10^{-7} \mathrm{M}, 10^{-9} \mathrm{M}$ and $10^{-11} \mathrm{M}$. 
To quantitatively compare and accurately evaluate the SERS activity of the different substrates, the enhancement factor $(\mathrm{EF})$, defined as the ratio of the normalized SERS intensity of each substrate at its limit of detection over the conventional Raman band intensity of R6G, was determined and the results are summarized in Table 3. The EF was calculated as previously reported in our published work, ${ }^{17}$ and the characteristic R6G peak located at $606 \mathrm{~cm}^{-1}$ was used to calculate the EFs using the following equation:

$$
\mathrm{EF}=\frac{I_{\text {SERS }}}{N_{a d s}} / \frac{I_{\text {bulk }}}{N_{\text {bulk }}}=\frac{I_{\text {SERS }} N_{b u l k}}{I_{b u l k} N_{a d s}}
$$

Where $I_{\text {bulk }}$ and $I_{\text {SERS }}$ are the intensities of the $606 \mathrm{~cm}^{-1}$ peak obtained from bulk conventional Raman spectra and SERS spectra, respectively; $N_{b u l k}$ and $N_{a d s}$ are the number of R6G molecules adsorbed on the Si wafer and on the pure Ag nanoparticles and Ag@Au hetero-architecture substrates within the laser spot. In order to determine $N_{b u l k}, 5 \mu \mathrm{L}$ of an R6G solution $\left(10^{-3} \mathrm{M}\right)$ was spread onto the Si wafer to obtain a spot with a diameter of $\sim 5 \mathrm{~mm}$, whilst $N_{a d s}$ was determined by drop-coating $5 \mu \mathrm{L}$ of an R6G solution $\left(1 \times 10^{-11} \mathrm{M}\right)$ onto pure Ag nanoparticles and Ag@ Au substrates to obtain a spot of the same diameter. Within the sample laser spot, the ratio of $N_{b u l k} / N_{a d s}$ was calculated to be $10^{8}$. A sample calculation for the EF of substrate Ag@Au8 was performed as follows: under the same acquisition parameters, the intensity for the $606 \mathrm{~cm}^{-1}$ peak for $10^{-3} \mathrm{M}$ R6G on Si wafer and for $10^{-11} \mathrm{M}$ R6G on substrate Ag@Au8 were 2601 and 561, respectively. The ratio of $I_{S E R S} / I_{\text {bulk }}$ was therefore calculated to be 0.216 , which equates to an EF of $2.16 \times 10^{7}$ for the sampled site on the Ag@ Au8 substrate.

Table 3 Enhancement factor of the four SERS substrates

\begin{tabular}{ccccc}
\hline \hline \multirow{2}{*}{ Sample } & $\begin{array}{c}\text { Test } \\
\text { site }\end{array}$ & $\begin{array}{c}\text { Enhancement factor } \\
(\mathbf{E F})\end{array}$ & $\begin{array}{c}\text { Average value of } \\
\text { EF }\end{array}$ & $\begin{array}{c}\text { Deviation from } \\
\text { average value of EF }\end{array}$ \\
\hline \multirow{2}{*}{ Ag } & $\# 1$ & $1.60 \times 10^{7}$ & $-6 \%$ \\
& $\# 2$ & $1.84 \times 10^{7}$ & $1.70 \times 10^{7}$ & $+8 \%$ \\
& $\# 3$ & $1.66 \times 10^{7}$ & $-2 \%$ \\
\hline \multirow{2}{*}{ Ag@Au4 } & $\# 1$ & $1.78 \times 10^{7}$ & $-7 \%$ \\
& $\# 2$ & $1.93 \times 10^{7}$ & $+1 \%$ \\
\hline Ag@Au8 & $\# 3$ & $2.02 \times 10^{7}$ & $+6 \%$ \\
& $\# 2$ & $2.16 \times 10^{7}$ & & $-7 \%$ \\
& $\# 3$ & $2.35 \times 10^{7}$ & $2.30 \times 10^{7}$ & $+1 \%$ \\
\hline
\end{tabular}




\begin{tabular}{|c|c|c|c|c|}
\hline \multirow{3}{*}{ Ag@Au12 } & $\# 1$ & $0.55 \times 10^{7}$ & & $-8 \%$ \\
\hline & $\# 2$ & $0.62 \times 10^{7}$ & $0.60 \times 10^{7}$ & $+3 \%$ \\
\hline & \#3 & $0.64 \times 10^{7}$ & & $+7 \%$ \\
\hline
\end{tabular}

It is known that drop-coating solutions on solid substrates often results in the "coffee ring" effect, whereby molecules accumulate on the outer rim of the circle. In light of this, it is likely that the concentration of R6G at the center of the drop-cast spot may be different to that at the edges, implying that the intensity of the SERS signals obtained from different test sites within the spot may differ depending on sampling location. In order to minimize sampling artefacts resulting from the "coffee ring" effect, we therefore, deliberately collected the spectra from the central region of the spot to avoid the potentially higher concentration of R6G at the circle's margins. As shown in Table 3, there is good agreement between the EF values obtained from the three test sites, with a deviation from the average EF of less than $10 \%$ across all substrates and sampling locations. The low \%deviation from the average EF for each tested site within the spot is indicative of the homogeneous distribution of R6G within the sampled area, and of the ability to consistently detect R6G from different areas of the tested region with minimal intensity changes. In addition, a second set of pure Ag and Ag@Au substrates was prepared following our two-step protocol, and the SERS measurements described above were repeated to confirm SERS signal reproducibility. The experimental data from the parallel test is shown in Table S1 (ESI), and the results obtained from the second batch of pure Ag and Ag@Au substrates displayed similar trends in EFs as those listed in Table 3 for the first set of substrates. EFs increased as the thickness of the Au layer in the Ag@Au substrates increased up to $8 \mathrm{~nm}$, then decreased as the Au film was further thickened to $12 \mathrm{~nm}$ in substrate Ag@Au12. In light of the similar EF trends observed in the parallel test, only one series of EF data is shown for pure Ag nanoparticle and Ag@Au hetero-architecture substrates.

The SERS enhancement commonly comes from a multitude of factors, such as the composition of the metal particles, particle size, distance between adjacent particles, irradiation condition and type of adsorbed molecule. As all acquisition parameters used in the SERS measurements were identical, our analysis focused on the effect of substrate morphology on the EFs. The effect of nanoparticle size on SERS intensity has been both theoretically and experimentally studied and reported by other groups. ${ }^{18-21}$ The size of metal nanoparticles is not only linked to the electromagnetic field at the particle's surface, but also to the scattering efficiency as well as to the number of molecules that can adsorb to its surface. Reports indicate that the SERS intensity increases with increasing particle size, provided the nanoparticle diameter were below $70 \mathrm{~nm}^{20}$ or $150 \mathrm{~nm}^{21}$ 
With a further increase in particle size, the increased excitation was offset by increased scattering and a decrease in the total number of adsorbed target molecules, potentially leading to a decrease in SERS intensity. The average particle size of Ag nanoparticles on Si wafer and of the Ag@Au4, Ag@Au8 and Ag@Au12 substrates was below 40 nm, potentially implying that the negative effect from heightened scattering and potential reduction in number of adsorbed molecules did not affect the substrates sufficiently enough to cause signal dampening. The observed SERS signal from substrate Ag@Au4 and Ag@Au8 therefore increased as the average particle size grew from 26 nm for Ag nanoparticles to $31 \mathrm{~nm}$ for $\mathrm{Ag} @ \mathrm{Au}$ hetero-architectures. Stamplecoskie ${ }^{20}$ and Zhao et al. ${ }^{21}$ reported a maximum SERS intensity ratio of 5 for $70 \mathrm{~nm}$ to $20 \mathrm{~nm}$ and for $150 \mathrm{~nm}$ to $50 \mathrm{~nm}$ sized Ag nanoparticles. The size of nanoparticles in our substrates varied from $26 \mathrm{~nm}$ to $38 \mathrm{~nm}$, therefore the estimated SERS intensity ratio of $38 \mathrm{~nm} \mathrm{Ag} @ \mathrm{Au}$ nanoparticles to $26 \mathrm{~nm} \mathrm{Ag}$ nanoparticles is believed to be below 5. It should be noted however, that the SERS signal of substrate Ag@Au12 was lower than for the other substrates.

The nature of the SERS effect is also strongly dependent on the chemical composition and morphology of the substrate material. Only a limited number of noble metal nanostructures (gold, silver, etc.) have the potential to produce large SERS enhancements. In metallic nanoparticle substrates or aggregated colloidal suspensions, the junctions between closely packed nanoparticles are termed "hot spots". Within these regions, efficient coupling of the localized surface plasmons of neighboring nanoparticles leads to an increase in the local electromagnetic field at the junction, which is chiefly responsible for the huge enhancements in Raman scattering of any probe or target molecule located therein. ${ }^{22-27}$

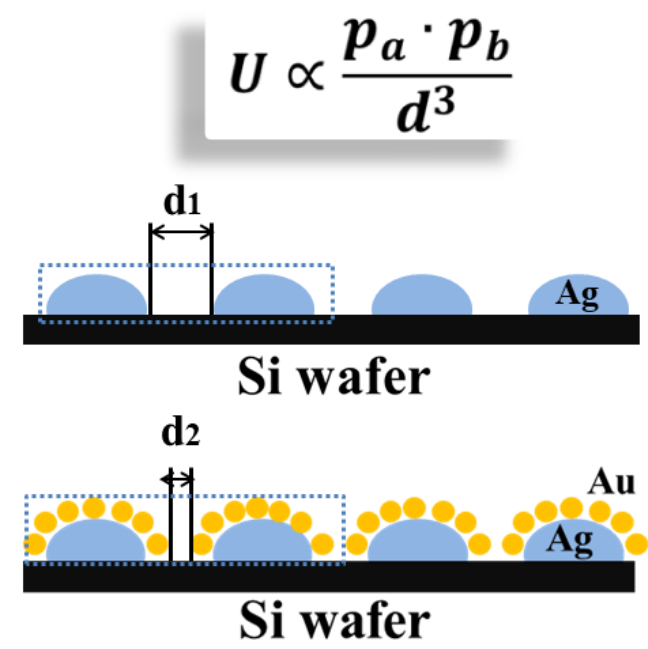

Fig. 5 Schematic of the mechanism for enhanced SERS intensity in Ag@Au hetero-architectures. 
In light of the above, it is believed that optimization of the gaps between adjacent nanoparticles in the substrate could lead to more efficient coupling of the localized surface plasmons and result in SERS signal enhancement. As shown in Fig. 5, when two neighboring Ag nanoparticles are coupled, the coupling energy $\mathrm{U}$ is inversely proportional to the third power of the distance $d$ (where the other parameters, $p_{a}$ and $p_{b}$, are the dipole moments of the two nanoparticles). ${ }^{24}$ As the gap between two adjacent nanoparticles becomes smaller, the coupling energy increases and the surface plasmon coupling effect is enhanced. It follows that the localized electromagnetic fields at the junction between nanoparticles will be strengthened, which in turn leads to an enhancement of the SERS intensity for species located within the hotspot. In the context of the $\mathrm{Ag} @ \mathrm{Au}$ hetero-architectures, coating Ag nanoparticles with Au films of defined thicknesses enables the efficient tuning of the gaps between adjacent nanoparticles, potentially leading to an enhancement of the SERS activity. In particular, when the distance between adjacent nanoparticles is below $10 \mathrm{~nm}$, Raman scattering is dramatically magnified. ${ }^{22,23,28,29}$

As shown in Fig. 2 and Table 1, the average distance between neighboring Ag nanoparticles decreased from $10.6 \mathrm{~nm}$ for pure $\mathrm{Ag}$ nanoparticles on $\mathrm{Si}$ wafer to $7.4 \mathrm{~nm}$ for the $\mathrm{Ag} @ \mathrm{Au} 8$ hetero-architecture substrate, implying that the SERS intensity of the hetero-architecture substrate should be greatly enhanced compared to the pure Ag nanoparticle substrate. Indeed, SERS measurements confirmed that the SERS signal for sample Ag@Au8 increased by 35\% relative to that of pure Ag nanoparticles on $\mathrm{Si}$ wafer. If the pure Ag nanoparticle substrate hypothetically possessed the same average size, size distribution and average gap size between adjacent particles as hetero-architecture sample Ag@Au8, approximated based on the expression in Fig. 5, the SERS signal should be enhanced by more than $35 \%$ because the average gap size in Ag@ Au8 was smaller than that between pure Ag nanoparticles on $\mathrm{Si}$ wafer. The limited enhancement observed for substrate Ag@Au8 might be attributed to the presence of the Au film. It is known that gold is less SERS active than silver; therefore, in spite of the potential SERS enhancement obtained by reducing the average gap between Ag nanoparticles by gold coating, the SERS signal enhancement is somewhat reduced by the presence of the gold film itself. As a result, milder overall SERS enhancement was observed for substrate Ag@Au8 due to the summation of the positive contribution to the SERS signal obtained by reducing the average gap between particles and of the negative contribution originating from the 
Au layer itself. The reduced SERS signal obtained from substrate Ag@Au12 may be attributed to the potential formation of a continuous Au film accompanied by a general decrease in the number of interparticle gaps, as seen in Fig. 2(d). Previous reports have demonstrated that hot spots contribute the most to the overall SERS signal enhancement. ${ }^{30-32}$ The presence of a flatter, more uniform substrate surface, with fewer gaps resulting from continuous Au film formation implies that fewer hot spots were present in substrate Ag@Au12. The number of R6G molecules located within a hot spot was therefore most likely reduced compared to the other tested substrates and would result in a decrease in the observed SERS signal. Meanwhile, the smaller average gap between particles would be less accessible to the probe molecule due to steric hindrance, also resulting in a net decrease in SERS signal intensity compared to the other substrates.

In practice, the ability to tune and achieve consistent control over the gaps between adjacent nanoparticles during the synthetic process is extremely challenging. To overcome this issue, we have proposed a two-step protocol for the synthesis of Ag@Au hetero-architectures which allows control over the final substrate morphology to be achieved by modulating the Au sputtering time. Furthermore, using this approach, hetero-architecture substrates displaying improved SERS sensitivity were successfully prepared, offering a straightforward way of producing highly sensitive SERS platforms for trace analyte detection.

\subsection{Chemical stability}

The chemical stability of the Ag and Ag@Au nanoparticle substrates in harsh environments was assessed using a similar method to that reported in previous studies. ${ }^{12,13,33}$ Briefly, the substrates were submerged in a $0.5 \mathrm{M} \mathrm{H}_{2} \mathrm{O}_{2}$ solution (oxidizer) for 1 hour at room temperature. Upon immersion of the pure Ag nanoparticle substrate in the $\mathrm{H}_{2} \mathrm{O}_{2}$ solution, an immediate strong reaction occurred, and a significant amount of small bubbles formed on the surface of the Ag nanoparticle substrate. After 3 minutes, the Ag nanoparticles were completely stripped from the substrate and the $\mathrm{H}_{2} \mathrm{O}_{2}$ solution produced no further bubbles. The typical reaction between $\mathrm{Ag}$ and $\mathrm{H}_{2} \mathrm{O}_{2}$ is given in equation (2). ${ }^{34}$

$$
\mathrm{Ag}_{\mathrm{n}}(\text { solid })+3 \mathrm{H}_{2} \mathrm{O}_{2}+2 \mathrm{H}^{+} \rightarrow \mathrm{Ag}_{\mathrm{n}-2}(\text { solid })+2 \mathrm{Ag}^{+}+\mathrm{O}_{2}+4 \mathrm{H}_{2} \mathrm{O}
$$

Other reports have also confirmed the gradual dissolution of $\mathrm{Ag}$ nanoparticles in solutions of 
$\mathrm{H}_{2} \mathrm{O}_{2}$ by monitoring the LSPR and observing its progressive dampening. ${ }^{35,36}$ In this work, confirmation of the absence of $\mathrm{Ag}$ nanoparticles on the $\mathrm{Si}$ wafer following immersion in $\mathrm{H}_{2} \mathrm{O}_{2}$ was obtained by examination of the substrate using FE-SEM. Upon immersion of the Ag@Au substrates in the $\mathrm{H}_{2} \mathrm{O}_{2}$ solution, fewer bubbles formed on the surface of the $\mathrm{Ag} @ \mathrm{Au}$ substrates compared to the pure Ag nanoparticle substrate, implying that the corrosive action of the oxidizer solution on the substrate was greatly suppressed by the presence of the Au coating. This work confirms previous reports that $\mathrm{Au}$ nanoparticles are more chemically inert to $\mathrm{H}_{2} \mathrm{O}_{2}$ than $\mathrm{Ag}$ nanoparticles, ${ }^{36}$ thus demonstrating that, for the Ag@Au hetero-architecture substrates the Au particle films effectively protect the $\mathrm{Ag}$ nanoparticles from corrosion by $\mathrm{H}_{2} \mathrm{O}_{2}$. Observation of the decreasing number of bubbles produced by the Ag@Au substrates indicated that the anti-corrosion protection offered by the $\mathrm{Au}$ films is proportional to the film thickness. The presence of thicker Au films severely limited the ability of $\mathrm{H}_{2} \mathrm{O}_{2}$ to react with the $\mathrm{Ag}$ nanoparticles on the $\mathrm{Si}$ wafer by forming a barrier, thereby effectively protecting them from dissolution. After immersion in the $\mathrm{H}_{2} \mathrm{O}_{2}$ solution for $1 \mathrm{~h}$, the average size of the particles on the Ag@Au substrates was very similar to that of the as-prepared substrates. However, more gaps were observed following immersion in $\mathrm{H}_{2} \mathrm{O}_{2}$ (see Fig. S2, ESI).

In order to quantify the degree of protection to the Ag nanoparticles provided by the Au films, the SERS spectra of R6G $\left(10^{-7} \mathrm{M}\right)$ on the pure Ag nanoparticle, Ag@Au4, Ag@Au8 and Ag@Au12 substrates were collected prior to, and following immersion in $\mathrm{H}_{2} \mathrm{O}_{2}$, and the intensity of $606 \mathrm{~cm}^{-1}$ peak was plotted as shown in Fig. 6. Before immersion in the $\mathrm{H}_{2} \mathrm{O}_{2}$ solution, the SERS intensity of R6G obtained from the different substrates (black line in Fig. 6) increased with increasing thickness of the Au film from 0 to $8 \mathrm{~nm}$, followed by a dramatic decrease at an Au film thickness of $12 \mathrm{~nm}$. As mentioned above, the enhanced SERS intensity observed for substrates Ag@Au4 and Ag@Au8 may be attributed to the enhanced surface plasmon coupling effect resulting from the optimal tuning of the gaps between adjacent Ag nanoparticles by deposition of the Au film. Conversely, the lower SERS signal acquired from Ag@Au12 is ascribed to the reduction in the number of "hot spots" between neighboring nanoparticles due to the formation of a flatter, continuous $\mathrm{Au}$ film over the $\mathrm{Ag}$ nanoparticles 


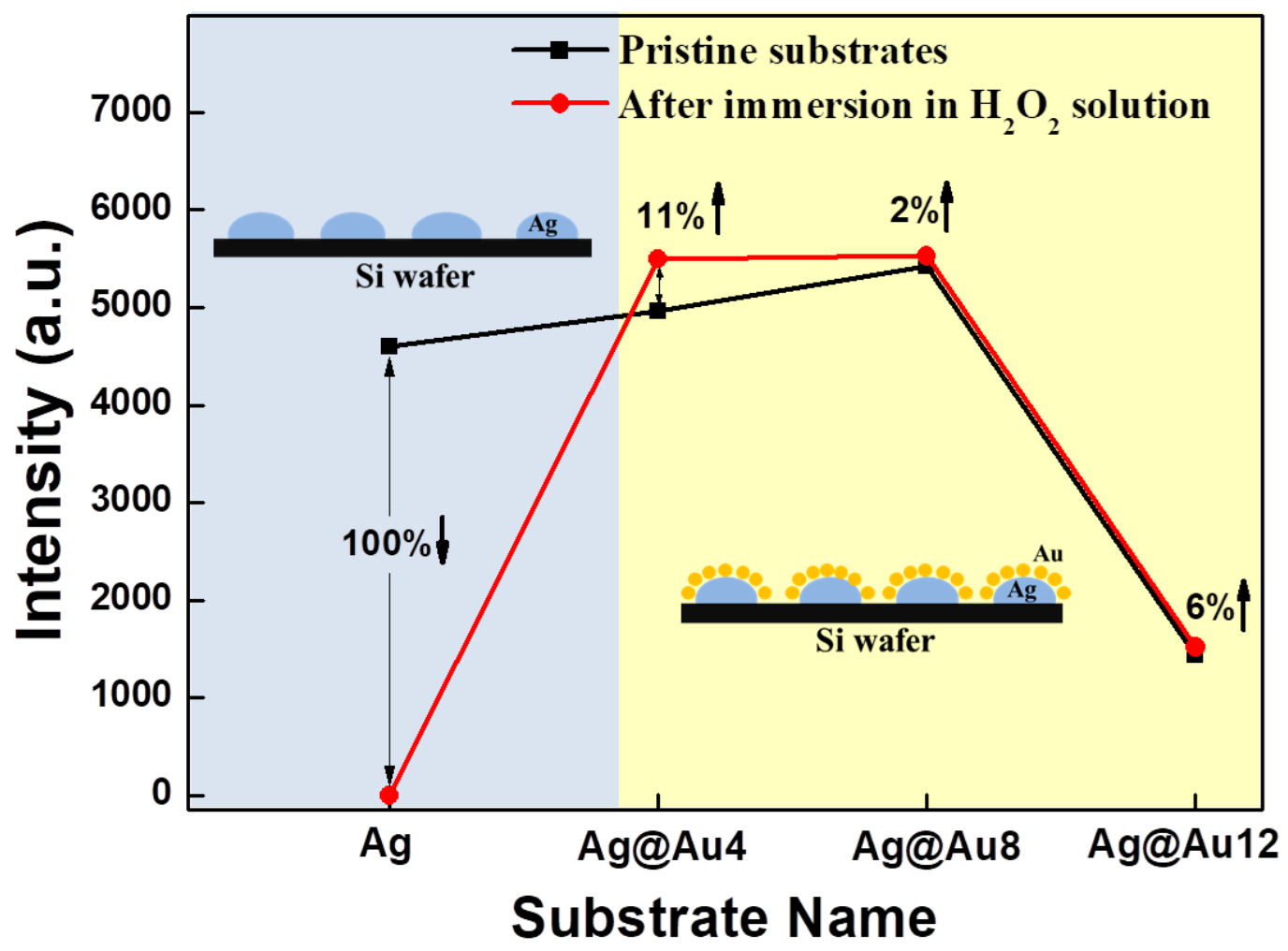

Fig. 6 Change in the SERS intensity of the $606 \mathrm{~cm}^{-1}$ peak of R6G $\left(10^{-7} \mathrm{M}\right)$ drop-coated on the Ag, Ag@Au4, $\mathrm{Ag} @ \mathrm{Au} 8$ and $\mathrm{Ag} @ \mathrm{Aul} 2$ substrates before and after immersion in a $0.5 \mathrm{M} \mathrm{H}_{2} \mathrm{O}_{2}$ solution.

Following immersion in the $\mathrm{H}_{2} \mathrm{O}_{2}$ solution, the SERS signal of the pure Ag nanoparticle substrate dropped to zero, due to the complete stripping of the nanoparticles from the Si wafer as a result of acid corrosion. In contrast, an overall increase in the SERS signal intensity of R6G was obtained from the other three Ag@Au substrates, with a percentage increase of 11\%, 2\% and 6\% for hetero-architecture substrate Ag@Au4,Ag@Au8 and Ag@Au12 respectively, as shown by the red line in Fig. 6. As mentioned above, progressive oxidation of $\mathrm{Ag}$ nanoparticles by $\mathrm{H}_{2} \mathrm{O}_{2}$ led to the complete dissolution of the $\mathrm{Ag}$ nanoparticles from the $\mathrm{Si}$ wafer in the absence of an $\mathrm{Au}$ film. However, based on the increase in SERS signal observed for the Ag@Au substrates, the decomposition of $\mathrm{Ag}$ nanoparticles by $\mathrm{H}_{2} \mathrm{O}_{2}$ was prevented by the presence of the protective $\mathrm{Au}$ films. Direct comparison of the SEM images of the Ag@Au substrates before (Fig. 2b, c, d) and after (Fig. $\mathrm{S} 2 \mathrm{a}, \mathrm{b}, \mathrm{c}$ ) immersion in the $\mathrm{H}_{2} \mathrm{O}_{2}$ solution revealed that substrate morphology was only minimally affected by the oxidizer. The slight changes in morphology of the hetero-architecture substrates are attributed to the reaction between $\mathrm{H}_{2} \mathrm{O}_{2}$ and the $\mathrm{Au}$ films, as confirmed by the formation of bubbles in solution. A further explanation for the increase in SERS signal observed following immersion of 
the substrates in $\mathrm{H}_{2} \mathrm{O}_{2}$ could be the formation of pinholes. Non-uniform $\mathrm{Au}$ film coverage over the $\mathrm{Ag}$ nanoparticles could have allowed a direct reaction between $\mathrm{Ag}$ and $\mathrm{H}_{2} \mathrm{O}_{2}$, leading to $\mathrm{Ag}$ nanoparticle dissolution and pinhole formation. However, the presence of small pinholes (at the scale of several nanometers) would not be detectable by FE-SEM. Hao et al. ${ }^{37}$ have used DDA calculations to show that the electric field enhancement due to pinholes can be 3-10 times larger than that generated by nanoshells, which is in agreement with the observed SERS signal enhancement from the Ag@Au substrates following immersion in $\mathrm{H}_{2} \mathrm{O}_{2}$. The potential pinholes formed due to the corrosion of the $\mathrm{Ag} @ \mathrm{Au}$ substrates in $\mathrm{H}_{2} \mathrm{O}_{2}$ act as "hot spots" that further enhance the SERS intensity, resulting in the overall increase in SERS signal observed across all Ag@Au substrates after immersion in $\mathrm{H}_{2} \mathrm{O}_{2}$. Furthermore, the SERS enhancement might be proportional to the density of the pinholes. The likelihood of pinhole formation by acid corrosion decreases as the thickness of the Au film increases, implying that both the number of pinholes and the exposed Ag area on Ag@Au12 would be smaller than for the other Ag@Au substrates. As a result, the largest SERS enhancement was observed for substrate Ag@Au4.

\section{Conclusions}

Ion sputtering was used to coat $\mathrm{Ag}$ nanoparticles on $\mathrm{Si}$ wafer with $\mathrm{Au}$ films of different thicknesses $(4,8$ and $12 \mathrm{~nm})$ in order to improve substrate chemical stability while maintaining high SERS sensitivity. FE-SEM images show that upon increasing the Au film thickness, the average particle size increased and the distance between adjacent Ag nanoparticles decreased accordingly. Successful tuning of the gaps between adjacent Ag nanoparticles led to SERS signal enhancement for substrates with Au films of $4 \mathrm{~nm}$ and $8 \mathrm{~nm}$, with substrate Ag@Au8 exhibiting the best SERS signal. The chemical stability of the substrates was evaluated by immersion in a $0.5 \mathrm{M} \mathrm{H}_{2} \mathrm{O}_{2}$ solution for $1 \mathrm{~h}$ with concomitant SERS signal evaluation. Pure Ag nanoparticles on Si wafer were completely dissolved by $\mathrm{H}_{2} \mathrm{O}_{2}$. Ag@ Au hetero-architecture substrates however, exhibited improved chemical stability as a result of the protective Au films. The SERS intensity changes before and after immersion of these substrates in $\mathrm{H}_{2} \mathrm{O}_{2}$ show that the enhanced chemical stability of the $\mathrm{Ag} @ \mathrm{Au}$ substrates is proportional to the thickness of the Au films. Based on both its SERS sensitivity and improved chemical stability, substrate Ag@Au8, with an Au film thickness of $8 \mathrm{~nm}$, is believed to represent the optimal candidate for SERS applications in oxidative environments or biological 
systems.

\section{Acknowledgement}

This work was supported by the President Fund and the Dean Fund from Xi'an Technological University (No.XAGDXJJ17001, No.2017GDYJZ01), the Open Fund of the Shaanxi Province Key Laboratory of Thin Films Technology and Optical Test (No.ZSKJ201705), and the Key Research \& Development Project of Shannxi Province (No. 2017GY-031). C.F. Wu also wishes to thank Xi'an Technological University for funding her stay in UK.

\section{Conflicts of Interest:}

The authors declare no conflict of interest.

\section{References}

1 S. Schlücker, Angew. Chem. Int. Ed., 2014, 53, 4756-4795.

2 D. K. Lim, K. S. Jeon, J. H. Hwang, H. Kim and J. Kwon, Nature Nanotech., 2011, 6, 452-460.

3 E. Chaffin, R.T. O'Connor, J. Barr, X. Huang and Y. Wang, J. Chem. Phys., 2016, 145, 054706.

4 X. Wang, G. Ma, A. Li, J. Yu, Y. Yang and L. Lin, Chem. Sci., 2018, 9, 4009-4015.

5 C. Wang, J. Fang, Y. Jin and M. Cheng, Appl. Surf. Sci., 2011, 259, 1144-1148.

6 Y. Cui, B. Ren, J Yao, R. Gu and Z.Q. Tian, J. Phys. Chem. B, 2006, 110, 4002-4006.

7 W. Xia, J. Sha, Y. Fang, R. Lu, Y. Luo and Y. Wang, Langmuir, 2012, 38, 5444-5449.

8 A. Gutés, R. Maboudian and C. Carraro, Langmuir, 2012, 28, 17846-17850.

9 P. Dong, Y. Lin, J. Deng and J. Di, ACS Appl. Mater. Interfaces, 2013, 5, 2392-2399.

10 G.V. Pavan Kumar, J. Raman Spectrosc., 2009, 40, 2069-2073.

11 L. Rivas, S. Sanchez-Cortes, J.V. García-Ramos and G. Morcillo, Langmuir, 2000, 16, 9722-9728.

12 N. Murshid, I. Gourevich, N. Coombs and V. Kitaev, Chem. Commun., 2013, 49, 11355-11357.

13 Y. Yang, J. Liu, Z.W. Fu and D. Qin, J. Am. Chem. Soc., 2014, 136, 8153-8156.

14 O. Prakash, S. Sil, T. Verma and S. Umapathy, J. Phys. Chem.C, 2020, 124(1), 861-869. 
15 C. Wu, E. Chen and J. Wei, Colloids Surf. A, 2016, 506,450-456.

16 L. Jensen and G. C. Schatz, J. Phys. Chem. A, 2006, 110, 5973-5977.

17 C. Wu, C. Cai, P. Yang and W. Liu, Colloids Surf. A, 2018, 545, 205-211.

18 V. N. Pustovit and T.V. Shahbazyan, Microelectronics J., 2006, 36, 559-563.

19 K. L. Kelly, E. Coronado, L. L. Zhao and G. C. Schatz, J. Phys. Chem. B, 2003, 107,668-677.

20 K. G. Stamplecoskie and J. C. Scaiano, J. Phys. Chem.C, 2011, 115, 1403-1409.

21 Y.Zhao, Y. Zhang, J. Meng, S.Chen, R. Panneerselvam, C.Li, S. b. Jamali, X. Li, Z. Yang, J. Li and Z. Tian, J. Ramn. Spectrosc., 2016, 47, 662-667.

22 E. C. Le Ru, E. Blackie, M. Meyer and P. G. Etchegoin, J. Phys. Chem. C, 2007, 111, 13794-13803.

23 C. Oubre and P. Nordlander, J. Phys. Chem. B, 2005, 109, 10042-10051.

24 I. Romero, J. Aizpurua, G. W. Bryant and F. J. García De Abajo, Opt. Express, 2006, 14, 9988-9999.

25 S.L.Kleinman, R.R.Frontiera, A.I.Henry, J.A.Dieringer and R.P.Van Duyne, Phys. Chem. Chem. Phys., 2013, 15, 21-36.

26 S. Sanchez-Cortes, J.V. Garcia-Ramos and G.J. Morcillo, J. Colloid Interface Sci.,1994,167, 428-436.

27 C.G. Blatchford, J.R. Campbell and J.A. Creighton, Surf. Sci., 1982, 120, 435-455.

28 H.Wang, L.Yao, X. Mao, K.Wang, L. Zhu and J. Zhu, Nanoscale, 2019, 11, 13917-13923.

29 J. Zhang, M. Irannejad and B. Cui, Plasmonics, 2015, 10, 831-837.

30 H. Lu, L. Zhu, Y. Lu, J. Su, R. Zhang and Y. Cui, ACS Appl.Mater.Interfaces, 2019, 11, 39359-39368.

31 Y. Fang, N. Seong and D. D. Dlott, Science, 2008, 18, 388-392.

32 S. Nie and S. R. Emory, Science, 1997, 275, 1102-1106.

33 Y. Zhang, C. Yang, B. Xue, Z. Peng, Z. Cao, Q. Mu and L. Xuan, Sci. Rep., 2018, 8, 898.

34 P. Miao, B. Wang, J. Yin, X. Chen and Y. Tang, Electrochem. Commun., 2015, 53, 37-40.

35 T. Parnklang, B. Lamlua, H. Gatemala, C. Thammacharoen, S. Kuimalee, B. Lohwongwatana and

S. Ekgasit, Mater. Chem. Phys., 2015, 153, 127-134.

36 P.J. Rivero, E. Ibañez, J. Goicoechea, A. Urrutia, I.R. Matias and F.J. Arregui, Sens. Actuators B, 2017, 251, 624-631. 
37 E. Hao, S. Li, R.C. Bailey, S. Zou, G.C. Schatz and J.T. Hupp, J. Phys. Chem. B, 2004, 108, 1224-1229. 